\title{
Preparation of monodispersed carboxylate-functionalized gold nanoparticles using pamoic acid as a reducing and capping reagent
}

\author{
Md. Abdul Aziz • Jong-Pil Kim • Munetaka Oyama
}

Published online: 8 February 2014

(C) The Author(s) 2014. This article is published with open access at SpringerLink.com

\begin{abstract}
A simple preparation method of gold nanoparticles (AuNPs) using pamoic acid (PA; 4,4'-methylene-bis(3-hydroxy-2-naphthalene carboxylic acid)) with $\mathrm{NaOH}$ is described. Although PA is insoluble in water, it can be dissolved in the presence of $\mathrm{NaOH}$ and function as a capping and reducing reagent to form the AuNPs. The thus-formed AuNPs have a good monodispersity with diameters of 10.8 $\pm 1.2 \mathrm{~nm}$ and carboxylate functions that come from the PA. Because PA is a methylene-bridged dimer of 3-hydroxy-2naphthalene carboxylic acid, the formation of the AuNPs was examined also using the analogous monomer molecules, i.e., 3-hydroxy-2-naphthalene carboxylic acid, 2-hydroxy-1-naphtalene carboxylic acid, or 2naphthol. Consequently, it was found that the case of PA was specific to forming the spherical monodispersed AuNPs while that differently shaped Au nanostructures were formed in the other cases. The present preparation using PA would be an interesting example that stable, monodispersed, carboxylate-functionalized AuNPs could be prepared without using thiols. Also, the present results may provide some
\end{abstract}

M. A. Aziz $(\square)$

Center of Research Excellence in Nanotechnology, King Fahd University of Petroleum and Minerals, Dhahran 31261, Saudi Arabia e-mail:maziz@kfupm.edu.sa

M. A. Aziz $\cdot$ M. Oyama $(\bowtie)$

Department of Material Chemistry, Graduate School of Engineering, Kyoto University, Nishikyo-ku, Kyoto 615-8520, Japan

e-mail: oyama.munetaka.4m@kyoto-u.ac.jp

\section{J.-P. Kim}

Surface Properties Research Team, Korea Basic Science Institute Busan Center, Busan 609-735, South Korea insight into molecular designs of capping reagents for preparing functionalized AuNPs without using thiol derivatives.

Keywords Hydrogen tetrachloroaurate · Pamoic acid · Carboxylate-functionalized gold nanoparticles $\cdot$ Capping reagent

\section{Introduction}

The chemical preparations of gold nanoparticles (AuNPs) have been attracting considerable attention due to their interesting properties and applicability in nanoscience and nanotechnology. As typical preparation methods of AuNPs in aqueous solutions, two approaches are well known, i.e., citrate ions have been used for the size-controlled synthesis of AuNPs [1] and tannic acid has been used for a rigid control of the size [2]. While a two-phase synthesis with thiol capping reagents [3] has been utilized for preparing AuNPs with smaller sizes and functional capping, the development of preparation methods of AuNPs in an aqueous solution is still active. The green synthesis of AuNPs $[4,5]$ would be one of the topics including the use of plants [6] and microorganisms [7]. In addition, preparation methods in which one reagent acts as a reducing and capping reagent have been proposed because of their simplicity [8-10].

When we consider the capping action of reagents toward AuNPs, in the case of the thiol derivatives, the Au-S bonding is a definite force for stabilizing the AuNPs. Various functional cappings have been performed with a variety of thiol 
derivatives. For example, carboxylate-functionalized AuNPs were prepared using some thiol derivatives [11-14]. On the other hand, in the cases of green synthesis as referred above, which were carried out in aqueous solutions, the formation of AuNPs is possible without forming any definite bonding between $\mathrm{Au}$ and the capping reagents. This means that, even though there are no peculiar groups to bond with the $\mathrm{Au}$, AuNPs can be prepared in aqueous solutions. Thus, to explore successful examples to form AuNPs would still be meaningful in order to know the molecular interactions to prepare AuNPs and apply the interactions to advanced syntheses of the AuNPs.

In the present paper, we show that pamoic acid (PA; 4,4'-methylene-bis(3-hydroxy-2-naphthalene carboxylic acid); 1) can work as an effective capping and reducing reagent in the presence of $\mathrm{NaOH}$ to form relatively monodispersed AuNPs. PA has two 2-naphthol units, which are bridged at position 1 of 2-naphthol by a methylene group, and each naphthol unit contains one carboxylic acid group at position 3 . While PA is usually utilized for the salt formation of some drugs [15], it was also used as a ligand for making dinuclear Ti(IV) complexes [16]. However, to the best of our knowledge, PA has never been used to prepare AuNPs.<smiles>O=C(O)c1cc2ccccc2c(Cc2c(O)c(C(=O)O)cc3ccccc23)c1O</smiles>

1

While we initially adopted PA as a trial, the use of PA has permitted us to prepare relatively monodispersed ca. 11-nm AuNPs at room temperature by working as a reducing and capping reagent in the presence of $\mathrm{NaOH}$. In addition, the carboxylate functions of the thus-formed AuNPs have been experimentally confirmed. Therefore, to explore the capping actions of PA, the formations of AuNPs were examined also using the analogous monomer molecules, i.e., 3-hydroxy-2naphthalene carboxylic acid (3H2NCA; 2), 2-hydroxy-1naphtalene carboxylic acid (2H1NCA; 3), and 2-naphtol (4).<smiles>O=C(O)c1cc2ccccc2cc1O</smiles>

2<smiles>O=C(O)c1c(O)ccc2ccccc12</smiles>

3<smiles>Oc1ccc2ccccc2c1</smiles>

4
Kundu and coworkers reported the use of 2-naphtol and 2,7-dihydroxy-naphthalene combined with UV or microwave irradiation for the interesting structural forming of $\mathrm{Au}$ or $\mathrm{Ag}$ nanocrystals [17-19]. While the naphthols have functions as reducing reagents in their studies, our results present that some naphthol derivatives have apparently less power for nanostructural production in comparison to PA.

The synthesis of AuNPs with a monodispersity under rigid size control have a significant scientific impact: successful size-controlled preparations have been reported for alkanethiol-protected AuNPs [20], and AuNPs were first prepared in an aqueous phase and then transferred to nonpolar solvents [21]. In our present trial, it would be characteristic that well-monodispersed AuNPs as in previous studies $[20,21]$ could be prepared with PA in an aqueous solution.

\section{Experimental}

Hydrogen tetrachloroaurate trihydrate $\left(\mathrm{HAuCl}_{4} \cdot 3 \mathrm{H}_{2} \mathrm{O}\right), \mathrm{PA}$, 3H2NCA, 2H1NCA, 2-naphthol, 3-aminopropyltrimethoxysilane (APTMS), and cadmium nitrate tetrahydrate were purchased from Sigma-Aldrich. All other reagents were purchased from Wako Pure Chemicals, Ltd. Indium tin oxidecoated glasses (ITO) were purchased from Geomatec Co., Ltd. (Yokohama, Japan). All solutions were prepared with ultrapure water obtained from a water purification system (Millipore WR600A, Yamato Co., Japan).

The UV-visible spectra were recorded by an optical spectrophotometer, USB 2000, Ocean Optics, Inc. The transmission electron microscopic and X-ray photoelectron spectroscopic analyses were performed at the Korea Basic Science Institute, Busan Center, Korea. The scanning electron 
microscopic (SEM) images were observed using a fieldemission scanning electron microscope (FE-SEM; JSM7400 F, JEOL, Japan).

As a typical preparation method of AuNPs with PA, $7.9 \mathrm{mg}$ PA was placed in a test tube and $9.0 \mathrm{ml}$ of pure water was added followed by sonication for $15 \mathrm{~min}$. Forty microliters of 1.0 $\mathrm{M} \mathrm{NaOH}$ (aq.) was then added to the solution, and pure water subsequently added to make the volume $10.0 \mathrm{ml}$. The mixture was then sonicated for $15 \mathrm{~min}$ to make the $2.0-\mathrm{mM}$ PA solution clear. Next, $100 \mu$ of $1.0 \mathrm{M} \mathrm{NaOH}$ (aq.) was added and sonicated for $1 \mathrm{~min}$; then, $10 \mathrm{ml}$ of a $1.34-\mathrm{mM}$ solution of $\mathrm{HAuCl}_{4}$ (aq.) was added under sonication and stored for $15 \mathrm{~min}$. Finally, the solution was stored for $60 \mathrm{~min}$ undisturbed to allow the complete formation of the AuNPs. We checked the necessity of this process by observing the changes in the absorption spectra and found that further stirring after the mixing tended to be unfavorable for preparing the monodispersed AuNPs. Similar preparations were carried out with other reagents, i.e., 3H2NCA, 2H1NCA, or 2naphtol, but, in these cases, the concentrations were increased to $4.0 \mathrm{mM}$, i.e., twice that of PA, to make the amount of the naphthol units the same.

In preparing some samples for the TEM and X-ray photoelectron spectroscopy (XPS) measurements, the prepared solution of the AuNPs with PA was centrifuged at 12,000 rpm and the obtained sediment was redispersed in $1 \mathrm{mM} \mathrm{NaOH}$ (aq.). The centrifugation and redispersion processes were repeated three times to remove any free or loosely bound molecules. For the TEM analysis, the AuNPs were transferred to a copper grid by dipping it into the purified alkaline solution of the AuNPs. For the XPS analysis, the purified alkaline solution of the AuNPs was dropped onto a cleaned ITO substrate and dried at $40{ }^{\circ} \mathrm{C}$.

In preparing the AuNP-modified ITO, a piece of ITO was immersed overnight in ethanol containing $2 \%$ APTMS $(v / v)$ at room temperature, and the amine-terminated ITO was prepared. After washing with ethanol, the electrode was dried by flowing nitrogen. On the other hand, the solution of the AuNPs was centrifuged at 12,000 rpm and the supernatant was decanted. The obtained sediment was redispersed in water. The centrifugation and redispersion processes were repeated three times. The APTMS-modified ITO was dipped in the purified aqueous AuNPs solution for $2 \mathrm{~h}$. After washing with water, the modified ITO electrode was dried at $40^{\circ} \mathrm{C}$.

\section{Results and discussion}

PA is insoluble in water, but its sodium salt can be dissolved. Therefore, in the present study, we first prepared a clear alkaline solution of $2.0 \mathrm{mM}$ PA by sonication followed by the further addition of $\mathrm{NaOH}$ to promote the PA function as a reducing reagent. The solution was then mixed with an aqueous solution of $\mathrm{HAuCl}_{4}$ under sonication, and the formation of AuNPs was initiated. After the mixing, the color of the solution changed from yellow to blackish and then deep red, which indicated the formation of the AuNPs. Figure 1a shows the absorption spectra and Fig. 1b (left) shows a photo of the thus-prepared solution. Since the alkaline solution of PA or $\mathrm{HAuCl}_{4}$ does not have any absorption in this wavelength region, the characteristic absorption peak observed at $507 \mathrm{~nm}$ in Fig. 1a and the color in Fig. 1b (left) can be attributed to the formation of the AuNPs. The absorption did not change with time, which could be recognized by the absorption spectrum recorded 7 days after the preparation of the solution (Fig. 1a). The color of the solution did not change over several months and no precipitates were formed. These results indicate the high stability of the synthesized AuNPs.

To confirm the capping states of the AuNPs by PA, we added a $0.15-\mathrm{ml}$ aqueous solution of $6.7 \mathrm{mM} \mathrm{Cd}\left(\mathrm{NO}_{3}\right)_{2}$, i.e., $\mathrm{Cd}^{2+}$, to a $1.35-\mathrm{ml}$ aqueous solution of the AuNPs, which was prepared by centrifuging and redispersing the AuNPs in water to remove any unbound PA. As a result, the color of the solution has changed to blue as shown in Fig. $1 \mathrm{~b}$ (right). It is known that such a color change is due to the aggregation of the AuNPs and that, when the carboxylate groups are present on the surfaces of the AuNPs, the aggregation proceeds in the presence of divalent cations, such as $\mathrm{Cd}^{2+}, \mathrm{Hg}^{2+}$, and $\mathrm{Pd}^{2+}$ [13]. Thus, it was proved that carboxylate groups are present on the surfaces of the present AuNPs prepared with PA. This would be reasonable judging from the molecular structure of PA having two carboxylate groups in one molecule. Because the carboxylate groups can be an anchor group to bind $\mathrm{Au}$
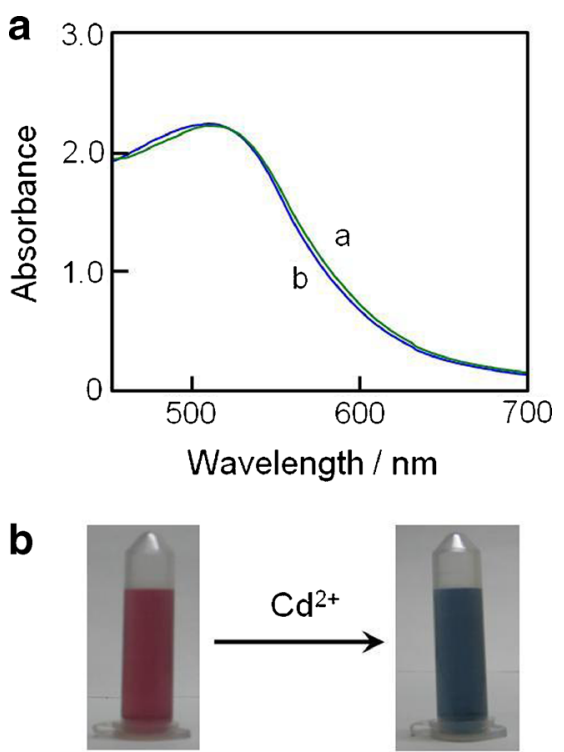

Fig. 1 a Visible absorption spectra of the solutions of AuNPs prepared with PA measured (a) just after the preparation and $(b)$ after 7 days. b A photo of a solution of AuNPs prepared with PA (left) and the color change after mixing with $\mathrm{Cd}^{2+}$ (right) 
Fig. 2 a A typical TEM image of AuNPs prepared with PA. b A high-magnification image of a. $\mathbf{c}$ A histogram depicting the size distribution of the AuNPs. d A typical FE-SEM image of AuNPmodified amine-terminated ITO surface
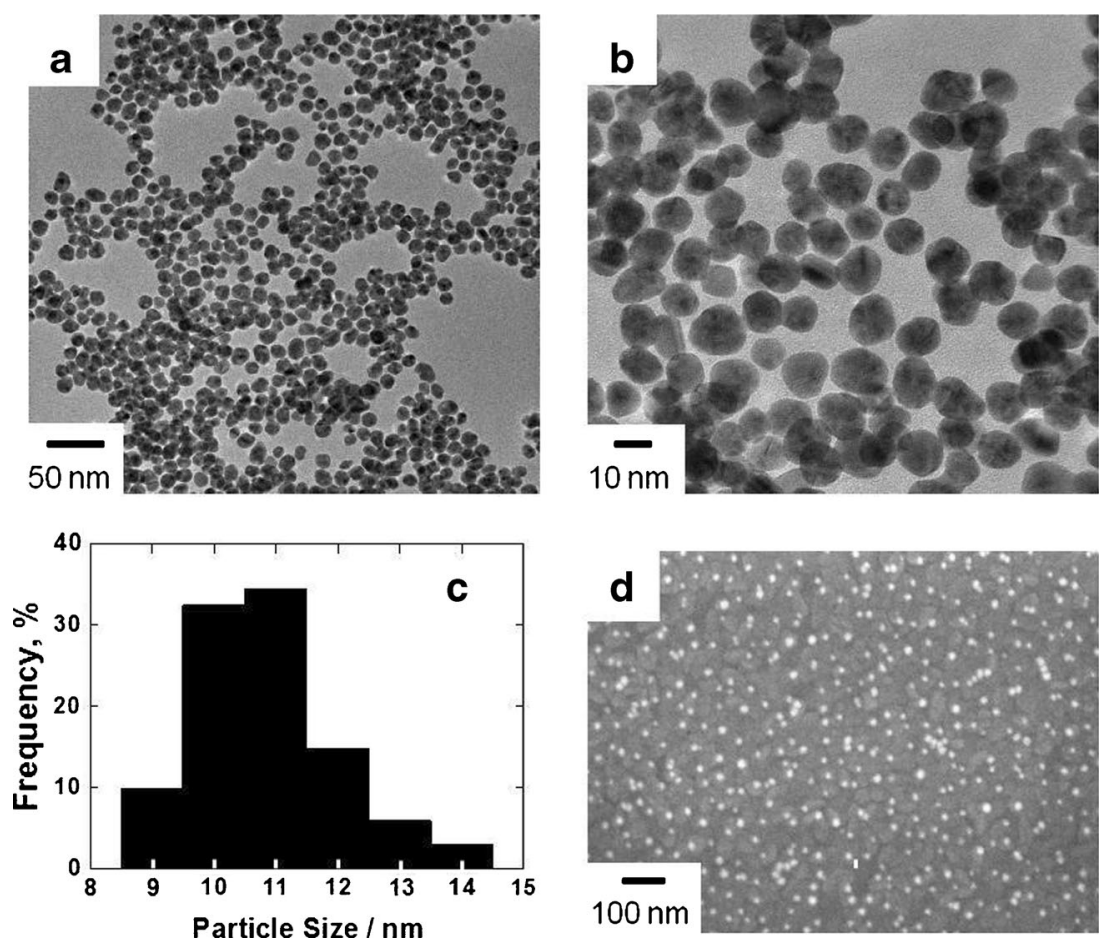

[22], it is considered that one of the carboxylate groups worked by contacting with $\mathrm{Au}$ and that the others located on the outer surface.

Next, we obtained TEM images of the AuNPs prepared with PA. The results are shown in Fig. 2a, b. The TEM images clearly indicate that the synthesized AuNPs are spherical and that the mean diameter was $10.8 \pm 1.2 \mathrm{~nm}$. The low standard deviation represents the good monodispersity of the prepared AuNPs. The histogram in Fig. 2c shows the size distribution. The diameters of the AuNPs are limited to between 8.5 and $14.5 \mathrm{~nm}$, and the diameters of $81.4 \%$ of the AuNP are in the range between 9.5 and $12.5 \mathrm{~nm}$. This dispersion was almost reproducible in several preparations under the same conditions. The good size regularity was also confirmed in the FESEM observation after immobilization of the AuNPs on amine-terminated ITO surfaces (Fig. 2d). We can see that the
AuNPs of the same size were homogeneously dispersed on the surface of the ITO. The attachment should be based on the electrostatic interaction between the amine groups terminated on the ITO surface and the carboxylate groups on each AuNP.

Furthermore, the present AuNPs prepared with PA were analyzed by XPS. The purified AuNPs showed only two peaks at 83.3 and 87.0 in Fig. 3a corresponding to the $\mathrm{Au}$ $4 f_{7 / 2}$ and $\mathrm{Au} 4 \mathrm{f}_{5 / 2}$ core-level binding energies which confirmed the presence of only metallic gold $\left(\mathrm{Au}^{0}\right)$ in the AuNPs [23]. In Fig. 3b, the $\mathrm{C} 1 \mathrm{~s}$ peaks were clearly identified at $284.6,288.6$, and $289.1 \mathrm{eV}$ which correspond to the $-\mathrm{C}-$ $\mathrm{C}-$, adsorbed $-\mathrm{COO}^{-}$group on the $\mathrm{Au}$ surfaces, and free $\mathrm{COO}^{-}$groups, respectively $[24,25]$.

As for the formation of the AuNPs with PA, it would be characteristic that the carboxylate-functionalized AuNPs can be easily prepared with a good monodispersity using PA as the
Fig. 3 a, b XPS spectra of AuNPs prepared with PA
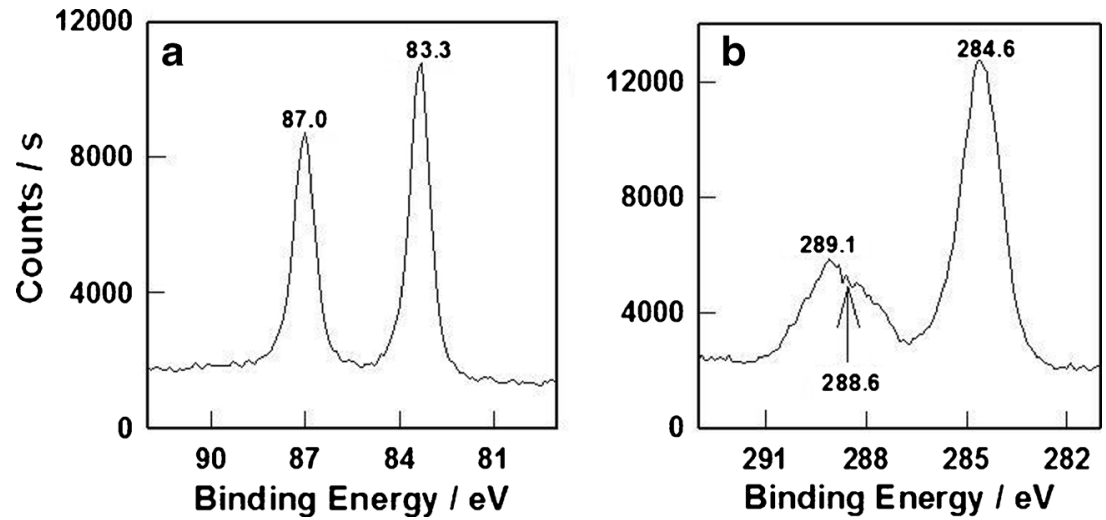
Fig. 4 a A typical TEM image of Au nanomaterials prepared with 2H1NCA. b A high-

magnification image of $\mathbf{a}$
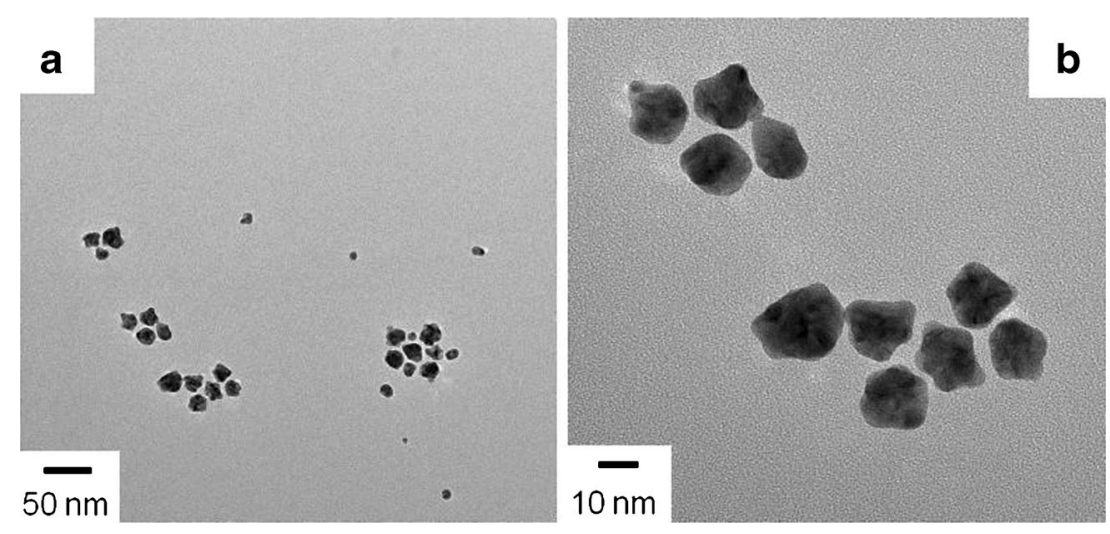

reducing and capping reagent. Because hydroquinone has been reported to have a function to reduce $\mathrm{AuCl}_{4}{ }^{-}$with $\mathrm{NaOH}$ [8], and because 2-naphtol derivatives work as a reducing reagent [17-19], the two hydroxyl groups of PA should be involved in the reduction of $\mathrm{AuCl}_{4}{ }^{-}$to form AuNPs. On the other hand, two carboxylate groups should have the key function for capping AuNPs and for characterizing the nature of the AuNPs by locating at the outer surface.

To compare the results obtained with PA, we next investigated the formation processes with 3-hydroxy-2-naphthalene carboxylic acid (3H2NCA; 2), 2-hydroxy-1-naphtalene carboxylic acid (2H1NCA; 3), and 2-naphtol (4). Consequently, we could never prepare monodispersed AuNPs as prepared with PA. For example, Fig. 4 shows the TEM results for AuNPs prepared with $2 \mathrm{H} 1 \mathrm{NCA}$. As apparently shown in the images, the shape of the AuNPs was not spherical but irregular, and the size dispersity was apparently not good. In this case, the absorption spectrum exhibited an absorption maximum at $558 \mathrm{~nm}$ (data not shown). Because the absorption maximum of the smaller AuNPs (ca. $10 \mathrm{~nm}$ ) should be around $510 \mathrm{~nm}$ as in Fig. 1a, the longer wavelength shift implied the formation of AuNPs with a larger size. In addition, the absorption spectrum changed to an absorption maximum at $541 \mathrm{~nm}$ after 7 days, which suggested that the stability of the AuNPs was not good in comparison to the AuNPs prepared with PA. This should be due to the weaker capping of
2H1NCA. Similar results were obtained with $3 \mathrm{H} 2 \mathrm{NCA}$; therefore, it is inferred that the position of a carboxylate group would not be very significant during the formation of the AuNPs. It should now be emphasized that the dimer structure of PA linked with a methylene group would be a key factor to form the monodispersed AuNPs, while the monomer may have a reducing power and weaker capping ability.

We also tried the same reduction of $\mathrm{AuCl}_{4}^{-}$with 2naphthol. As a result, the reduction processes of $\mathrm{AuCl}_{4}{ }^{-}$and the formed nanostructures of gold were totally changed in comparison to the other cases. Actually, after mixing with the solution of $\mathrm{HAuCl}_{4}$, the color of the solution turned dark gray and the color was maintained after a 15 -min sonication. Figure 5 shows TEM images of the formed materials. Some strange-shaped nanowires, like worms, were formed together with small nanospheres. The diameter of the nanowires was as small as $3.3 \pm 0.5 \mathrm{~nm}$. In a previous report, the gray color of the solution has been noted for gold nanochains [26]. Therefore, the present color change matched well with the formation of the nanowires. The smaller nanostructure as in Fig. 5 might be a reflection of the lower reduction power and the unique capping of 2-naphtol. However, the lack of the carboxylate is inferred to be unsuitable for capping; therefore, other capping molecules might be necessary as in the previous studies [17-19].
Fig. 5 a A typical TEM image of Au nanowires prepared with 2naphthol. b A high-magnification image of a
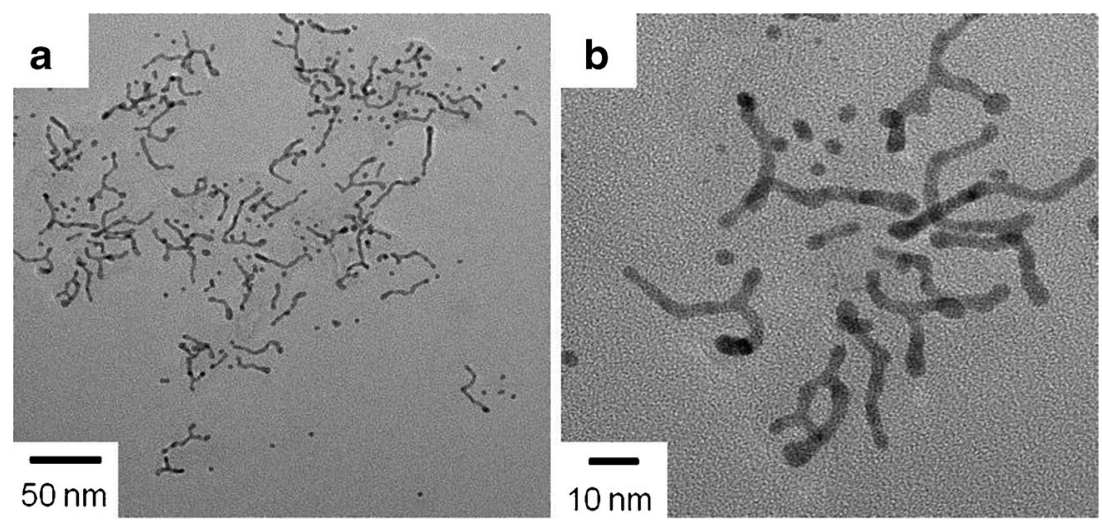
Based on the previous results obtained with $3 \mathrm{H} 2 \mathrm{NCA}$, 2H1NCA, and 2-naphtol instead of PA, we concluded that one naphthalene moiety of the capping reagents was not sufficient to stabilize the formed AuNPs, though they reduced the $\mathrm{AuCl}_{4}^{-}$. Therefore, it is inferred that the rigid size control (or capping) with PA has been permitted by the methylenelinked two naphthalene moieties. In addition, based on the formation of the wormlike Au nanowires with 2-naphtol and the ill-shaped AuNPs with 3H2NCA and 2H1NCA, the carboxylate group should have the function to stabilize the AuNPs. Thus, the rigid capping of PA is regarded to come from the methylene-linked naphthalene moieties and two carboxylate groups located outside.

\section{Conclusions}

During the reduction of $\mathrm{AuCl}_{4}{ }^{-}$with $\mathrm{PA}$ in the presence of $\mathrm{NaOH}$, monodispersed AuNPs, with diameters of 10.8土 $1.2 \mathrm{~nm}$, could be easily prepared. Reflecting on the presence of two carboxylate groups in PA, the formed AuNPs have carboxylate functions because only one carboxylate group would be used in the capping interaction. While the anchoring power of a carboxylate group is normally weak [22], due to the help of the naphthalene moieties of PA, it is expected that effective capping could be achieved while maintaining the monodispersity. As for the effects of aromatic rings, some integration with gold have previously been reported [27], and hydroquinone has been reported to form AuNPs [8]. As one remarkable achievement of the present study, the stable, monodispersed, carboxylate-functionalized AuNPs could be prepared without using thiols, while the known carboxylatefunctionalized AuNPs had been prepared with thiol anchors [11-14]. Thus, the proposed facile synthesis in an aqueous solution should be worthwhile reporting as a safe and clean synthesis of carboxylate-functionalized AuNPs.

In our trials using the analogous monomer molecules instead of PA, it was found that structural controls were difficult in spite of some progress in the reductions of $\mathrm{AuCl}_{4}^{-}$. Judging from the formation of the ill-shaped Au nanowires with 2naphtol, one carboxylate group would be helpful for nanostructural construction. However, the key factors of the rigid size control with PA would be due to the methylenelinked naphthalene moieties and two carboxylate groups. The present results may provide some information about molecular designs for carboxylate-functionalized AuNPs without using thiol derivatives.

Furthermore, in a previous study, heat treatment has been reported to be effective for the precise size control of alkanethiol-protected AuNPs [20]. Also, for the aqueousphase synthesis of AuNPs to be transferred to nonpolar solvents [21], the ratio of borohydride anions/hydroxyl anions was a key factor for the size control. While our present study has been limited to the preparation of AuNPs of $10.8 \pm 1.2 \mathrm{~nm}$ in aqueous solutions at room temperature, we are going to explore the possibility of the size control with PA as the next step.

Acknowledgments M. A. A. thanks the Japan Society for the Promotion of Science (JSPS) for the fellowship. This work was supported by JSPS KAKENHI Grant Numbers 20550074 and 21-09245.

Open AccessThis article is distributed under the terms of the Creative Commons Attribution License which permits any use, distribution, and reproduction in any medium, provided the original author(s) and the source are credited.

\section{References}

1. Frens G (1973) Nature Phys Sci 241:20-22

2. Slot JW, Geuze HJ (1985) Eur J Cell Biol 38:87-93

3. Brust M, Walker M, Bethell D, Schiffrin DJ, Whyman R (1994) Chem Commun 801-802

4. Raveendran P, Fu J, Wallen SL (2006) Green Chem 8:34-38

5. Wu CC, Chen DH (2007) Gold Bull 40:206-212

6. Kumar V, Yadav SK (2009) J Chem Technol Biotechnol 84: $151-157$

7. Das SK, Marsili E (2010) Rev Environ Sci Biotechnol 9:199-204

8. Sirajuddin MA, Torriero AAJ, Nafady A, Lee CY, Bond AM, O'Mullane AP, Bhargava SK (2010) Colloids Surf A 370:35-41

9. Wu CC, Chen DH (2010) Gold Bull 43:234-240

10. Badwaik VD, Bartonojo JJ, Evans JW, Sahi SV, Willis CB, Dakshinamurthy R (2011) Langmuir 27:5549-5554

11. Chen S, Kimura K (1999) Langmuir 15:1075-1082

12. Yonezawa T, Kunitake T (1999) Colloids Surf A 149:193-199

13. Kim Y, Johnson RC, Hupp JT (2001) Nano Lett 1:165-167

14. Lin YC, Yu BY, Lin WC, Lee SH, Kuo CH, Shyue JJ (2009) J Colloid Interface Sci 340:126-130

15. Jørgensen M (1998) J Chromatogr B 716:315-323

16. Baghel GS, Rao CP (2009) Polyhedron 28:3507-3514

17. Kundu S, Panigrahi S, Praharaj S, Basu S, Ghosh SK, Pal A, Pal T (2007) Nanotechnology 18:075712

18. Kundu S, Peng L, Liang H (2008) Inorg Chem 47:6344-6352

19. Kundu S, Wang K, Liang H (2009) J Phys Chem C 113:134-141

20. Shimizu T, Teranishi T, Hasegawa S, Miyake M (2003) J Phys Chem B 107:2719-2724

21. Martin MN, Basham JI, Chando P, Eah SK (2010) Langmuir 26: $7410-7417$

22. Chen F, Li X, Hihath J, Huang Z, Tao N (2006) J Am Chem Soc 128: 15874-15881

23. Aziz MA, Patra S, Yang H (2008) Chem Commun: 4607-4609

24. Han SW, Joo SW, Ha TH, Kim Y, Kim K (2000) J Phys Chem B 104: 11987-11995

25. Briggs D, Brewis DM, Dahm RH, Fletcher IW (2003) Surf Interface Anal 35:156-167

26. Polavarapu L, Xu QH (2008) Nanotechnology 19:075601

27. Syomin D, Kim J, Koel BE, Ellison GB (2001) J Phys Chem B 105: 8387-8394 